\title{
Design and Test of a Prototype Cavity for a Stern-Gerlach Polarimeter
}

\author{
P. Cameron ${ }^{1}$, M. Conte ${ }^{2}$, N. D’Imperio ${ }^{1}$, W. Franklin ${ }^{3}$, D.A. Goldberg ${ }^{4}$, A. Luccio ${ }^{1}$, W.W.

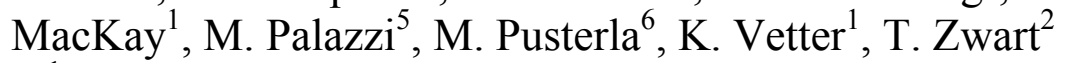 \\ ${ }^{1}$ Brookhaven National Laboratory, Upton NY 11973 USA \\ ${ }^{2}$ Universita and Sezione INFN di Genova, 16146 Genova, Italy \\ ${ }^{3}$ MIT-Bates Laboratory, Boston MA 01949 USA \\ ${ }^{4}$ Lawrence Berkeley National Laboratory, Berkeley, CA 94720 USA \\ ${ }^{5}$ AMS Naval Systems, Via Hermada 6/B, 16154 Genova, Italy \\ ${ }^{6}$ Universita and Sezione INFN di Padova, 35131 Padova, Italy
}

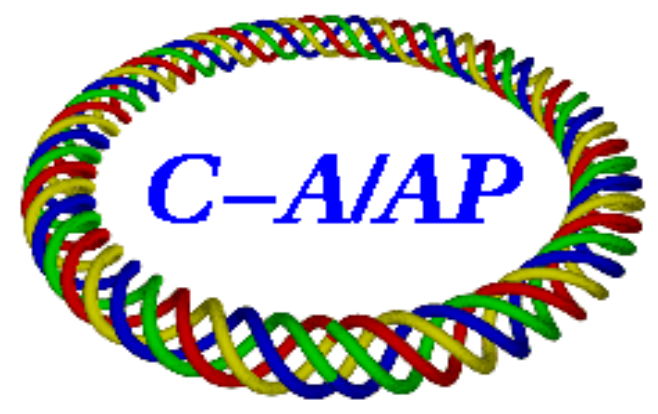
Collider-Accelerator Department
Brookhaven National Laboratory
Upton, NY 11973




\title{
DESIGN AND TEST OF A PROTOTYPE CAVITY FOR A STERN-GERLACH POLARIMETER*
}

\author{
P. Cameron ${ }^{1}$, M. Conte ${ }^{2}$, N. D’Imperio ${ }^{1}$, W. Franklin ${ }^{3}$, D.A.Goldberg ${ }^{4}$, A. Luccio ${ }^{1}$, W.W. MacKay ${ }^{1}$, M. Palazzi ${ }^{5}$, \\ M. Pusterla ${ }^{6}$, K. Vetter ${ }^{1}$, T. Zwart ${ }^{2}$ \\ ${ }^{1}$ Brookhaven National Laboratory, Upton, NY 11973, USA \\ ${ }^{2}$ Universita and Sezione INFN di Genova, 16146 Genova, Italy \\ ${ }^{3}$ MIT-Bates Laboratory, Boston MA 01949 USA \\ ${ }^{4}$ Lawrence Berkeley National Laboratory, Berkeley, CA 94720 USA \\ ${ }^{5}$ AMS Naval Systems, Via Hermada 6/B, 16154 Genova, Italy \\ ${ }^{6}$ Universita and Sezione INFN di Padova, 35131 Padova, Italy
}

\begin{abstract}
We present the design, simulations and measurements of a prototype passive RF cavity for a polarimeter with a transversely polarized electron beam interacting with the longitudinal gradient of the transverse component of magnetic field in the TE011 mode of the cavity. Magnetic coupling of a polarized electron beam at the Bates accelerator may produce signals of the order of a microwatt, permitting fast and accurate polarization measurement.
\end{abstract}

\section{INTRODUCTION}

Historically, beam polarization measurement has been a scattering experiment, with the attendant complexity of target and detector installation and operation. In addition, in the regimes currently of interest, analyzing powers are small, and often not accurately known. As a result, accurate polarization measurements are difficult, and slow in comparison with measurement of other beam parameters. This has consequences both for the efficiency of accelerator operations and the quality of physics data. The goal of the effort described in this paper is to accomplish fast and accurate polarization measurement not with a scattering experiment, but rather with conventional beam instrumentation.

The essence of the problem is twofold. First, the interaction of the magnetic moment with a beam pickup is appallingly small. Means must be employed to enhance this interaction. Second, the interaction of the electric charge with a beam pickup is appallingly large. Means must be employed to deal with the dynamic range problem, to accomplish the magnetic moment measurement in the presence of the electric charge background.

We have at hand two means to contend with the smallness of the magnetic moment. First, there is some sense in which the moment has geometry, and consequently is not a relativistic invariant. For instance (and perhaps somewhat counter-intuitively), the longitudinal moment as seen from the lab frame increases linearly with the relativistic gamma factor[1]. This effect was utilized in earlier efforts to design a polarimeter[2]. These efforts were confounded by the circumstance that (with the exception of non-linear devices, like squids) one must utilize time-varying electromagnetic fields to obtain interaction between beam and pickup, and the $\gamma$ dependent interaction of the moment with the space and time gradients of the magnetic field in the pickup are of opposite sign, so that to order $1 / \gamma$ these interactions cancel. Following this realization, considerable effort led to the discovery[3] of a pickup mode in which there is a $\gamma^{2}$ dependence in the interaction of the transverse moment with the field in the pickup. This is a bit surprising. While the transverse moment is a relativistic invariant, its interaction with this particular pickup mode is not.

The second means of contending with the smallness of the magnetic moment is much less esoteric than the first, and consists of simply resonating the relevant pickup mode to enhance the interaction. Our intent is to resonate a rectangular cavity in the TE011 (to measure vertical polarization) and TE101 (to measure horizontal) modes at frequencies close to the $2.856 \mathrm{GHz}$ RF fundamental of the MIT-Bates storage ring[4].

Given that means have been employed to render measurable the appallingly small moment, the next task is to deal with the dynamic range problem resulting from the relative largeness of the electric charge. There are two ways in which the dynamic range problem manifests. The first is the overlap of the tails of the adjacent TM modes, which are strongly excited by the beam charge, onto the TE signal modes. This requires that the $\mathrm{Q}$ of the TM modes be as large as possible to minimize energy in the tails, and causes the option of mode damping to appear unattractive. In addition, some method of TM mode suppression may be required.

The second is the direct excitation of the TE signal modes by the beam charge. Here again we may take advantage of the fact that the moment has geometry, that for the moment there exists an additional degree of freedom beyond those available to the charge. In the MIT-Bates ring the measurement is favored by the presence of a single full snake. The consequent reversal of spin direction from turn to turn results in locating the spectral line due to polarization at the half-integer, midway between the revolution lines, a clean location in the spectrum. The additional dynamic range that can be obtained by this separation in frequency domain is of the order of $100 \mathrm{~dB}$. Furthermore, slight detuning of the snake will split this line, the amount of splitting being 
proportional to the detuning. This will provide a clean spectral signature that is unique for the beam polarization.

\section{SIGNAL MODE POWER}

The magnetic moment signal power[3,7] expected from the cavity polarimeter TE011 mode at MIT-Bates is

$$
\frac{d U}{d t}=\frac{4 Q f\left(\mu \tau n \gamma^{2} P\right)^{2}}{\pi \varepsilon_{0} c^{2} V}
$$

where $\mathrm{Q}=5000, \mathrm{f}=2.856 \mathrm{GHz}$ is the bunching frequency, $\varepsilon_{0}$ is the permittivity of free space, $\mathrm{V} \sim 500 \mathrm{~cm}^{3}$ is the cavity volume, $\mu$ is the magnetic dipole moment of the electron, $\tau \sim 0.7$ is the transit time factor, $n=10^{8}$ is the number of electrons per bunch for all buckets filled and $50 \mathrm{ma}$ beam current, $\gamma=2000$ is the relativistic gamma factor at $1 \mathrm{GeV}, \mathrm{P} \sim 0.6$ is the beam polarization, and $\mathrm{c}$ is the speed of light. Evaluation of equation (1) shows that the polarization signal power expected at MIT-Bates will be about 100 microwatts, or $-40 \mathrm{dBm}$. This is a whopping big signal. In comparison, Schottky signals are typically at $-140 \mathrm{dBm} / \mathrm{Hz}$ or less, and thermal background is $174 \mathrm{dBm} / \mathrm{Hz}$.

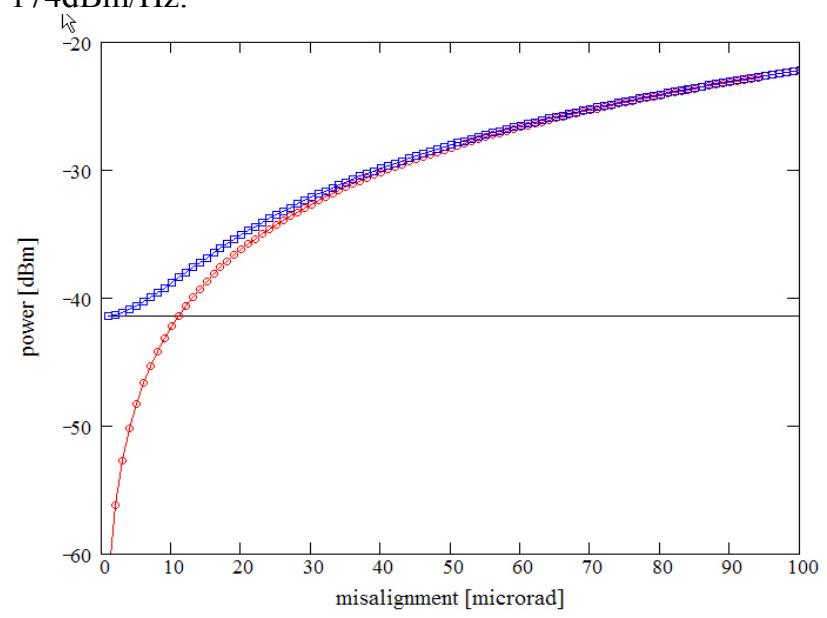

Figure 1 - Moment (straight line at $\sim-44 \mathrm{dBm}$ ) and charge (circles/red line) powers and their sum (squares/blue line) as a function of beam/cavity alignment.

In addition to being driven by the magnetic moment, the TE011 signal mode will be driven by the beam charge. In theory there is no longitudinal electric field associated with TE modes, and the Panofsky-Wentzel theorem[5,6] tells us that there can then be no coupling to the charge. However in practice this requires perfect alignment of beam and pickup. In practice, the electric charge signal power expected from the same mode is

$$
\frac{d U}{d t}=\frac{2 Q f(q \tau n d(\sin \phi))^{2}}{\pi \varepsilon_{0} V}
$$

where $d=7 \mathrm{~cm}$ is the cavity length, and $\phi$ is the angular misalignment of the beam axis relative to the cavity axis. Figure 1 shows moment and charge power as a function of cavity alignment. Moment power is a weak function of alignment accuracy, whereas charge power is a strong function. As previously mentioned, the presence of a full snake in the MIT-Bates Ring places the moment power at the half integer, whereas the charge power appears at the revolution line. The difference in their alignment behavior will provide an additional means to discriminate between TE011 mode excitation due to beam polarization and that due to beam charge.

\section{TM MODE POWER}

There are two conclusions which can be drawn at this point. First, the small interaction of the magnetic moment with the pickup can be enhanced sufficiently to easily permit observation. And second, with the combination of beam alignment and placement of the moment signal in a clean portion of the spectrum, this enhanced TE mode signal is not overwhelmed by the electric charge interaction. The last major point which must be considered is the interaction of the beam charge with TM cavity modes, and the overlap of those modes with the TE signal modes.

Table 1 shows frequencies and relative strengths of the TE011 mode and the various TM modes for the untrimmed prototype cavity, without beampipes. Of the eighteen TM modes listed here, the coupling of twelve to the beam is minimized by beam centering in the cavity. The remaining six will contribute strongly to the longitudinal impedance seen by the beam. This requires further attention from the perspective of machine stability, and may require that the machine be run with lower-than-usual beam current. From the point of view of the polarization signal strength, this is not a problem. Of the six modes that couple strongly to the beam, all except two couple weakly to the pickup probe. Those two modes

\begin{tabular}{|c|c|c|c|c|c|}
\hline $\begin{array}{l}\text { freq } \\
\text { [GHz] }\end{array}$ & mode & $\begin{array}{l}\text { Ex at } \\
\text { probe }\end{array}$ & effect of probe position & $\begin{array}{l}\text { Ez at } \\
\text { beam }\end{array}$ & effect of beam position \\
\hline 2.382 & TM110 & $0.0 \mathrm{E}+00$ & position of probe in $z$ only & 7.1E+02 & weak \\
\hline 2.724 & TE011 & $6.4 \mathrm{E}+02$ & weak & $0.0 \mathrm{E}+00$ & weak \\
\hline 3.203 & TM111 & 7.5E+02 & weak & $9.6 \mathrm{E}+02$ & weak \\
\hline 3.766 & TM120 & $0.0 E+00$ & position of probe in $y$ and $z$ & $0.0 E+00$ & position of beam in $y$ \\
\hline 3.766 & TM210 & $0.0 E+00$ & position of probe in z only & $0.0 E+00$ & position of beam in $x$ \\
\hline 4.332 & TM121 & $0.0 E+00$ & position of probe in y only & $0.0 E+00$ & position of beam in $y$ \\
\hline 4.332 & TM211 & $1.0 \mathrm{E}+03$ & weak & $0.0 \mathrm{E}+00$ & position of beam in $x$ \\
\hline 4.764 & TM220 & $0.0 \mathrm{E}+\infty 0$ & position of probe in $y$ and $z$ & $0.0 E+00$ & position of beam in $x$ and $y$ \\
\hline 4.900 & TM112 & $0.0 \mathrm{E}+00$ & position of probe in $z$ only & $1.5 \mathrm{E}+03$ & weak \\
\hline 5.223 & TM221 & $0.0 \mathrm{E}+\infty$ & position of probe in y only & $0.0 E+00$ & position of beam in $x$ and $y$ \\
\hline 5.326 & TM130 & $0.0 \mathrm{E}+00$ & position of probe in $z$ only & $4.8 \mathrm{E}+03$ & weak \\
\hline 5.703 & TM122 & $0.0 E+\infty$ & position of probe in $y$ and $z$ & $0.0 E+00$ & position of beam in $y$ \\
\hline 5.703 & TM212 & $0.0 \mathrm{E}+\infty$ & position of probe in $z$ only & $0.0 \mathrm{E}+00$ & position of beam in $x$ \\
\hline 5.740 & TM131 & 4.1E+03 & weak & $5.2 \mathrm{E}+03$ & weak \\
\hline 6.073 & TM230 & $0.0 \mathrm{E}+\infty 0$ & position of probe in z only & $0.0 E+00$ & position of beam in $x$ \\
\hline 6.406 & TM222 & $0.0 \mathrm{E}+\infty 0$ & position of probe in $y$ and $z$ & $0.0 \mathrm{E}+00$ & position of beam in $x$ and $y$ \\
\hline 6.439 & TM231 & $4.5 \mathrm{E}+03$ & weak & $0.0 \mathrm{E}+00$ & position of beam in $x$ \\
\hline 6.834 & TM132 & $0.0 \mathrm{E}+00$ & position of probe in z only & $6.1 \mathrm{E}+03$ & weak \\
\hline 7.431 & TM232 & $0.0 \mathrm{E}+00$ & position of probe in z only & $0.0 E+00$ & position of beam in $x$ \\
\hline
\end{tabular}
are highlighted in red.

We can divide equation (1) by equation (2) to find a scaling factor for relative power between the charge and moment interactions.

$$
\text { ratio }=2 \cdot\left(\frac{\mu \gamma^{2} P}{q d c}\right)^{2} \cong 10^{-10}
$$

The charge interaction is about $100 \mathrm{~dB}$ greater than the moment interaction. Assuming that the nearest and most 
dangerous TM111 mode has a Lorentzian lineshape, this shape is described by

$$
A(\omega)=\frac{1 / \delta \omega_{0}}{1+\left(2 \cdot\left(\omega-\omega_{0}\right) / \delta \omega_{0}\right)^{2}}
$$

where $\mathrm{A}(\omega)$ is the amplitude at the frequency $\omega, \omega_{0}$ is the resonance frequency, and $\delta \omega_{0}$ is the resonance width. Relative power (amplitude squared) for this lineshape is plotted below for the TM111 mode.

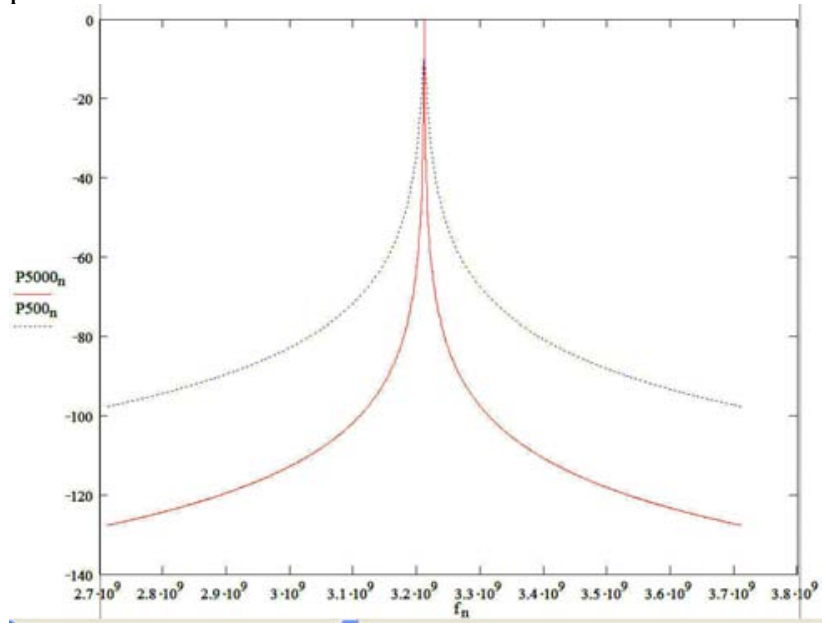

Figure 2 - Lorentzian lineshape for $\mathrm{Q}=5000$ (red curve) and $\mathrm{Q}=500$ (blue curve)

The horizontal scale of this figure is $\mathrm{Hz}$, and the vertical is $\mathrm{dB}$ relative to the peak of the $\mathrm{Q}=5000$ resonance. From this figure it is clear that, despite the fact that power is $10 \mathrm{~dB}$ higher for the red curve $(\mathrm{Q}=5000)$ on resonance, as one moves off resonance the power in the tails quickly dominates for the blue curve $(\mathrm{Q}=500)$. Power at the $\sim 2.8 \mathrm{GHz}$ frequency of the TE011 signal mode due to the TM111 mode is down by $\sim 120 \mathrm{~dB}$. From equation (3) the charge interaction exceeds the moment interaction by $\sim 100 \mathrm{~dB}$, so that we can expect the moment signal at the TE011 mode frequency to be $\sim 20 \mathrm{~dB}$ above the tail of the undamped TM111 mode.

\section{THE PROTOTYPE CAVITY}

A prototype cavity has been fabricated for the purpose of exact determination of cavity dimensions and frequencies (including the beampipe perturbation), and to determine probe lengths for optimal coupling.

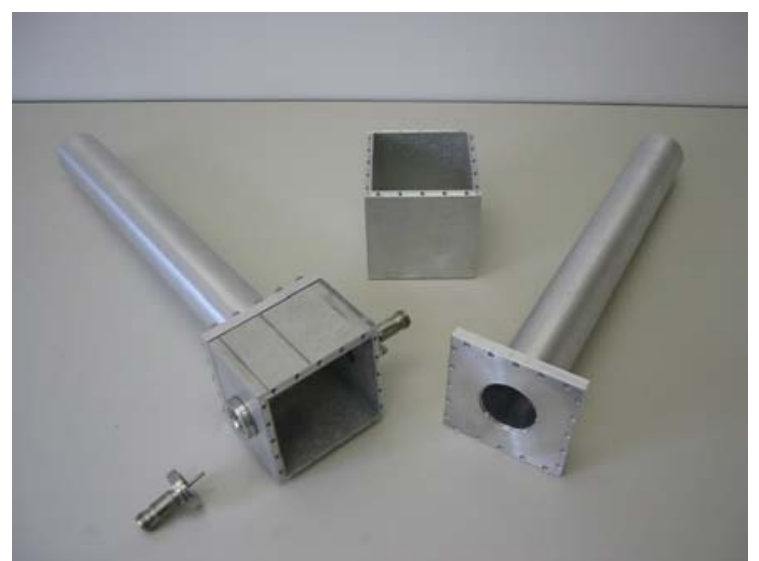

Figure 3 - The prototype cavity

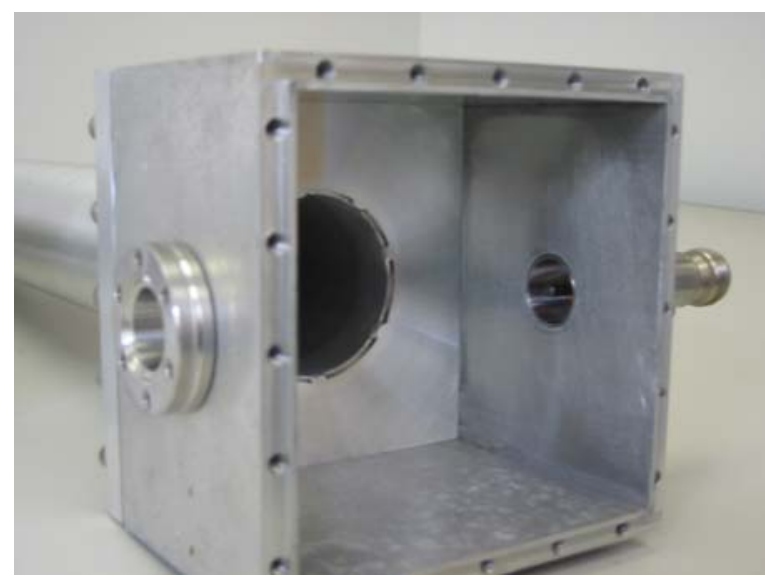

Figure 4 - Zoom showing probe detail

The cavity body was machined from commercially available square aluminum tubing. Initial cavity dimensions were $8.9 \mathrm{~cm} \times 8.9 \mathrm{~cm} \times 7 \mathrm{~cm}$. The TE011 mode probes are commercially available N-type feedthrus, mounted on mini-CF flanges. Figures 3 and 4 show details of the cavity and probes.

S21 transmission measurements through the two probes were completed on this cavity, both with the ends blanked off and with the beampipes installed. The results of these measurements are shown in figures 5 and 6 . The mode frequencies (see Table 1) for the closed box were calculated both analytically[8] and with in-house finite difference software[9], and are in good agreement with the observed frequencies. 
Bates Prototype S21 comparison - closed box and beampipe

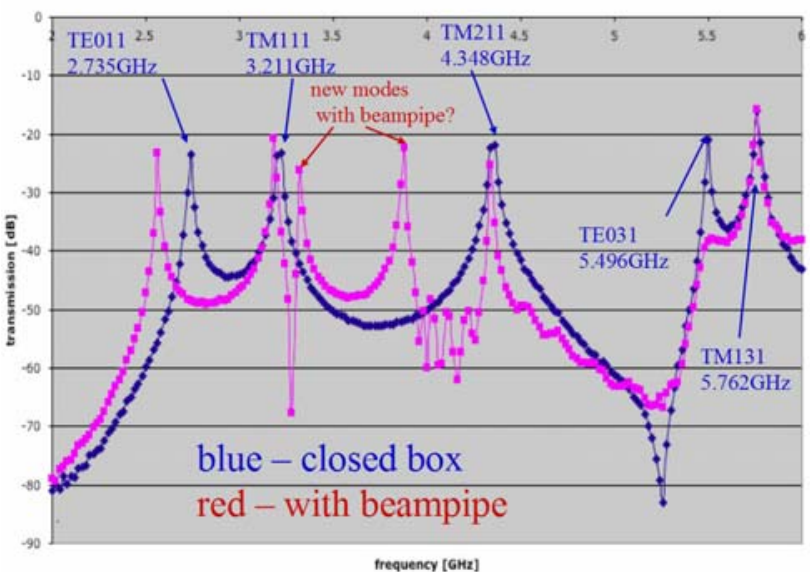

Figure 5 - S21 measurements of the prototype

As can be seen from Table 1, in our measurements we expect to see transmission only in those modes with nonzero transverse electric field at the probe locations. From the third column of the table, we see that corresponds to the TE011 and TM111/211/131 modes. These modes are identified in figure 5, as well as a TE031 mode not shown in the table. With the addition of the beampipes the TM mode frequencies were perturbed only slightly, where as the TE011 mode was shifted significantly and the TE031 mode was strongly damped. Two new modes also appeared. These modes are not understood, and are under investigation. Measured Q's with beampipes in place were $\sim 2500$, considerably less than the expected $\sim 5000$. We are aware of several possible causes of this reduced Q. First, the probe length was not yet adjusted for optimal coupling. Second, feedthru construction is not optimized for low loss. And finally, joints in the Aluminum cavity construction were not designed to minimize resistance.

Based on the measurement results shown in figure 5, a cavity length was calculated[9] to bring the TE011 mode closer to the $2.856 \mathrm{GHz}$ RF frequency. The cavity was trimmed to this length, and measurement results on the trimmed cavity are shown in figure 6 .

Bates Prototype S21 after trim

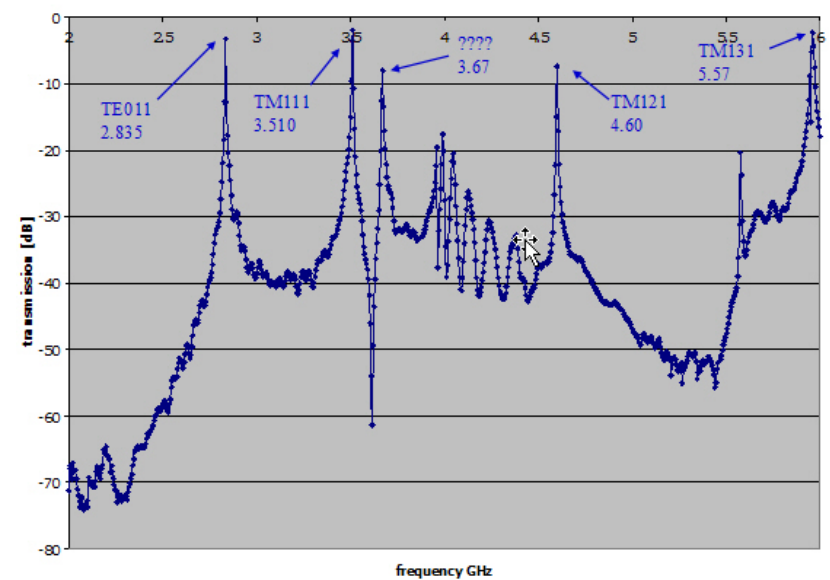

Figure 6 - S21 measurement of the trimmed prototype

As can be seen by the figure, all mode frequencies were shifted significantly by the trim, and one of the two unidentified modes that appeared when the beampipes were added seems to be diminished by some sort of interference effect.

\section{DATA ACQUISITION}

Figure 7 shows the intended data acquisition scheme. This scheme is simple and straightforward, and requires the purchase of a few inexpensive off-the-shelf components.

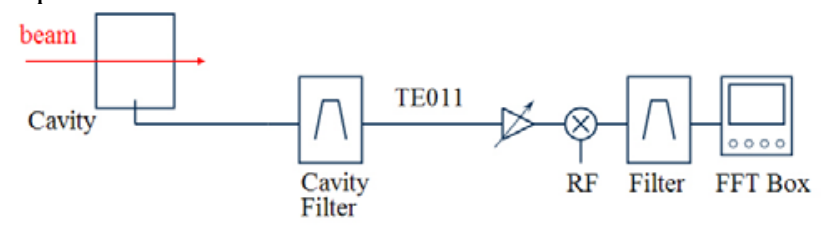

Figure 7 - Data Acquisition

Signal from one of the coupling probes passes through a non-vacuum cavity filter whose dimensions are proportioned to reject all modes except TE011. The cable length from the cavity to the filter might also be adjusted such that one or more of the reflected TM modes arrive back at the pickup cavity anti-phased to the beam excitation, resulting in some mode supression.

A slightly more sophisticated scheme of mode suppression is shown in figure 8 , where a non-vacuum cavity filter of dimensions identical to those of the pickup cavity is inserted before the TE011 mode filter cavity. It is possible to couple strongly to the TM modes in this cavity, but only weakly to the TE signal modes. One or more feedback loops can then be driven to suppress some or all of the TM modes.

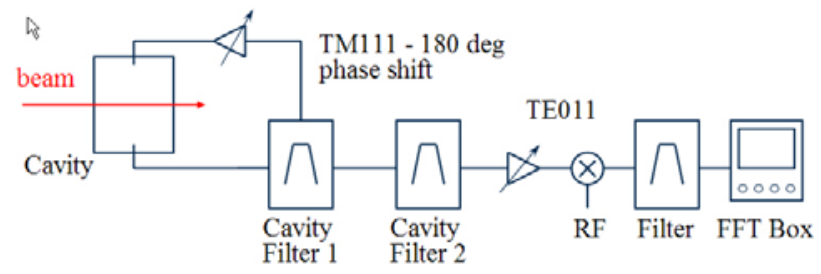

Figure 8 - Mode Supression

While this mode suppression is probably not required for the MIT-Bates effort, an additional goal is to explore possibilities for suppression of the charge interaction at RHIC.

\section{THE PROPOSED EXPERIMENT}

There exists the possibility to install a polarimeter cavity at MIT-Bates in early May of this year. A companion document to this paper, describing the accelerator configuration and operation for this experiment, is in preparation. Our intent for this experiment is to fabricate a simple (slightly) rectangular indium-soldered copper cavity, with the lower TE011 mode tuned 1.5 revolution lines below the $2.856 \mathrm{GHz}$ RF fundamental, and the upper TE101 mode tuned 1.5 revolution lines above the RF fundamental. Initial data acquisition will be as shown in Figure 7. 


\section{POLARIMETER AT RHIC}

With successful completion of the MIT-Bates experiment, one might consider installing the same cavity in RHIC. The situation is much more difficult there, where both $\gamma$ and the relevant magnetic moment are much smaller. The first consideration would be that there is little or no longitudinal bunch structure in RHIC at the cavity frequency. For this we suggest to install in RHIC a longitudinal bunching cavity at the polarimeter cavity frequency. With a $1 \%$ modulation of the longitudinal envelope, and performing the calculation of equation (1) for conditions to be found at RHIC ( 55 bunches, $10^{11}$ per bunch, $50 \%$ polarization, $\gamma=266, \mathrm{Q}=5000$ ), the resulting signal power is about $-130 \mathrm{dBm}$. Again, this is a good strong signal relative to Schottky signals. The problem is to observe this signal in the presence of the charge background. Similar to the discussion of Sections 2 and 3, the problem separates into excitation of the TE signal modes by the beam charge, and overlap of the tails of adjacent TM modes onto the TE signal modes.

Considering first excitation of the signal modes by the beam charge, Figure 9 is the equivalent of Figure 1 for RHIC parameters. With cavity and beam aligned to 1 microradian, the excitation of the TE011 mode by the beam charge is slightly less than $80 \mathrm{~dB}$ above the excitation by the magnetic moment. However, if one takes advantage of the additional spin degree of freedom, this spin signal can be separated from the charge signal, and at least in principle can be measured. Given sufficient dynamic range in the preamplifier, and mixing to audio frequencies to permit filtering and to provide sufficient bits in the digitizer, this measurement is possible. In addition, it may be possible to adjust cavity dimensions to take advantage of a $1 / \gamma^{2}$ suppression of the charge interaction due to transit time effects[3]. This requires further study.

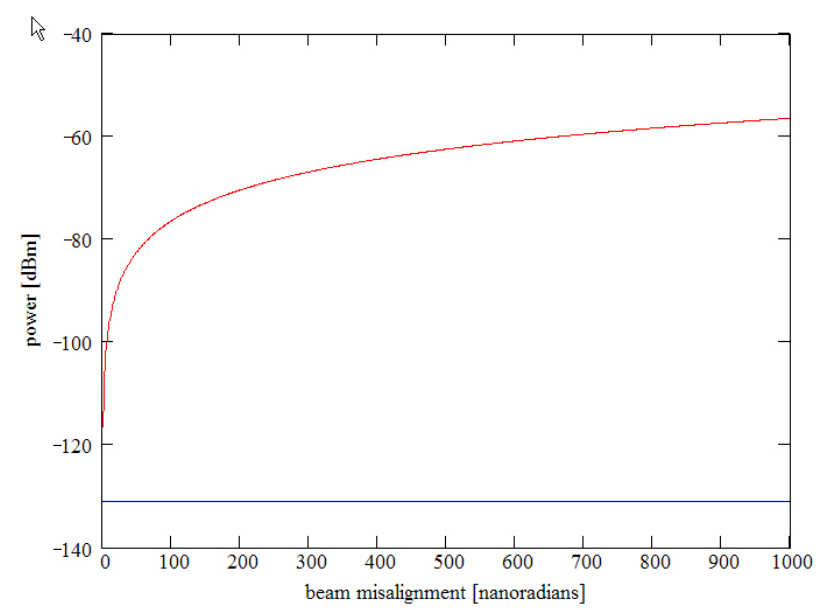

Figure 9 RHIC Moment (straight line at $\sim-130 \mathrm{dBm}$ ) and charge (red line) powers as a function of beam/cavity alignment.

Considering now overlap of the tails of adjacent TM modes, evaluation of equation (3) for RHIC parameters shows that the charge interaction is about $200 \mathrm{~dB}$ greater than the moment interaction. The first $100 \mathrm{~dB}$ of this is taken out by the Lorentzian lineshape. Some additional suppression, perhaps by the methods discussed in section 5 , will be required to reduce the dynamic range sufficient to permit observation of the spin lines between the revolution lines.

In any case, to perform this measurement at RHIC will be exceedingly delicate, and if attempted will require strong preliminary development work in the more favorable conditions found at MIT-Bates.

\section{CONCLUSION}

The present collaboration has at least three clearly defined goals. The first is to verify that there exists $\gamma^{2}$ dependence in the interaction of the beam magnetic moment with the selected cavity mode. The second is to utilize this interaction in the realization of fast and accurate polarimetry, first at MIT-Bates, and then possibly at RHIC. The third, given the $\gamma^{2}$ scaling, is to promote the possibility of implementing the reciprocal device, the spin-splitter, for the purpose of providing polarized proton beams at the LHC. The second iteration of prototype cavity measurements has been completed, and fabrication of the final vacuum-compatible cavity has begun. Our intent is to install this cavity at MIT-Bates early in May of this year.

\section{REFERENCES}

[1] J.D. Jackson, Classical Electrodynamics $2^{\text {nd }}$ Ed., Wiley, p557.

[2] P.R. Cameron et al, "An RF Resonance Polarimeter Phase 1 Proof-of-Principle Experiment", AGS/RHIC/SN 57. (1998).

[3] M. Conte et al, "The Stern-Gerlach Interaction between a travelling particle and a time varying magnetic field" http://xxx.lanl.gov/list/physics/0003, preprint 0003069

[4] T. Zwart et al, "Polarized Electrons in the MIT-Bates South Hall Ring", PAC 2001, Chicago.

[5] W.K.H. Panofsky and W.A. Wentzel, RSI 27, 967 (1956).

[6] D.A. Goldberg and G.R. Lambertson, "Dynamic Devices: A Primer on Pickups and Kickers", AIP 249, 539 (1992).

[7] P.R. Cameron et al, "Proposal for a Cavity Polarimeter at MIT-Bates", PAC 2001, Chicago.

[8] R.E. Collin, Foundations for Microwave Engineering $\underline{2}^{\text {nd }}$ Ed., McGraw-Hill, p500.

[9] N. D’Imperio, MAXSSIM. 
